\title{
Genetic Diversity and Population Structure of Rhododendron canescens, a Native Azalea for Urban Landscaping
}

\author{
Lav K. Yadav \\ Horticulture Department, University of Georgia, Athens, GA 30602
}

\author{
Edward V. McAssey \\ Institute of Breeding, Genetics, and Genomics, University of Georgia, \\ Athens, GA 30602
}

\author{
H. Dayton Wilde ${ }^{1}$ \\ Horticulture Department, University of Georgia, Athens, GA 30602; and \\ Institute of Breeding, Genetics, and Genomics, University of Georgia, \\ Athens, GA 30602
}

\begin{abstract}
Additional index words. genotyping-by-sequencing, ornamental plant, Piedmont azalea, single nucleotide polymorphism
\end{abstract}

\begin{abstract}
Rhododendron canescens is a deciduous azalea native to the southeastern United States that is used in landscaping due to its ornamental qualities. A genotyping-bysequencing (GBS) approach was taken to characterize the genetic structure and diversity of a $R$. canescens germplasm collection. Single nucleotide polymorphisms (SNPs) were identified by two software platforms, STACKS and GBS-SNP-CROP. Three distinct $R$. canescens populations were detected by STRUCTURE analysis with GBS-SNP-CROP data, whereas two populations were distinguished using STACKS data. Principal component analysis (PCA) with data from both SNP pipelines supported the presence of three populations. Statistical results indicated that there was low genetic differentiation between the populations, but relatively high genetic diversity within populations. The inbreeding coefficient of the $R$. canescens accessions was low, which would be expected with an outcrossing species. These results suggest that there may be a significant level of gene flow between populations of $R$. canescens.
\end{abstract}

Rhododendron canescens (Michaux) Sweet is a deciduous shrub commonly known as Piedmont azalea or sweet azalea. It is a diploid species $(2 n=26)$ that has a native range in the southern United States from Texas to North Carolina. $R$. canescens is a member of the Rhododendron section Pentanthera, a group of interfertile species that generally maintain species identity through habitat preference and flowering time. Twelve species of section Pentanthera are native to regions of the southern United States. Where R. canescens populations overlap with other native azalea species, hybridization and introgression have been demonstrated (King, 1977; Kron et al., 1993).

$R$. canescens is of value as an ornamental landscaping plant due to its showy, scented flowers; wide geographic distribution; and lace bug resistance (Galle, 1967; Wang et al.,

\footnotetext{
Received for publication 28 Dec. 2018. Accepted for publication 23 Jan. 2019.

This work was supported by Specialty Crop Block Grant 16-SCBGP-GA-0010, the Azalea Society of America, and the U.S. Department of Agriculture National Institute of Food and Agriculture Hatch project GEO00755. We thank Matthew Chappell for advice and Carol Robacker, Ron Miller, and Charles Andrew for providing azalea material.

${ }^{1}$ Corresponding author. E-mail: dwilde@uga.edu.
}

1998). It is one of the first native azaleas to bloom and could benefit native pollinators in urban landscapes in early spring (Mader et al., 2011). Cultivars of $R$. canescens and $R$. canescens hybrids are available currently for a niche market. $R$. canescens has architectural characteristics that may limit more widespread use in urban settings, namely an open growth habit and height up to $5 \mathrm{~m}$. We are interested in looking for genetic variation in wild germplasm that could be used to develop a more compact phenotype for landscaping.

Genetic analysis of species in Rhododendron section Pentanthera has been conducted using DNA sequences of the internal transcribed spacer region of rRNA genes (Scheiber et al., 2000) and the chloroplast $m a t K$ and $t r n K$ intron region (Kurashige et al., 2001). These studies found little sequence variation among species of the section Pentanthera, suggesting that they are a closely related group. Low genetic diversity among section Pentanthera species was further observed in a study with several hundred amplified fragment length polymorphism (AFLP) markers (Chappell et al., 2008). In that study, AFLP analysis was also used to investigate variation between and within populations of individual azalea species, including $R$. canescens. In contrast to the low level of variation found between populations and species, a high level of variation was observed within populations. Variation within $R$. canescens populations may be due in part to hybridization and introgression from other native azalea species (Chappell et al., 2008).

We obtained leaf samples from $290 R$. canescens genotypes, with a long-term objective of screening for variation in genes known to control plant height and branching. We first investigated the genetic diversity of a representative subset of this collection using GBS. Through GBS analysis, thousands of SNP markers can be generated and used to examine genetic diversity in nonmodel species (Peterson et al., 2014). We used SNP genotypes to characterize the genetic structure and diversity of the $R$. canescens germplasm collection.

\section{Materials and Methods}

Plant material collection. Young leaves were collected from $247 R$. canescens plants from 18 sites across Georgia, primarily within the Piedmont ecoregion (Omernik and Griffith 2014). Plants sampled at each site were at least $10 \mathrm{~m}$ apart and the species was confirmed based on floral characteristics as described by Kron (1993). The GPS coordinates of the plants were recorded, and the samples were frozen and stored at $-80{ }^{\circ} \mathrm{C}$ until further use. In addition, silica-dried leaves from $43 R$. canescens plants were received from collaborators in Georgia and northern Florida. The locations of the accessions used for GBS analysis are shown in Supplemental Table 1.

GBS library preparation and sequencing. Approximately $150 \mathrm{mg}$ of frozen leaf tissue was ground using a TissueLyser bead mill (Qiagen, Valencia, CA), and DNA was isolated using an E.Z.N.A. HP Plant DNA kit (Omega Bio-Tek, Norcross, GA), following the manufacturers' protocols. The DNA quantity was measured with a Qubit 2.0 (Invitrogen, Carlsbad, CA) using a Qubit dsDNA HS assay kit. DNA quality was determined by analysis with a NanoDrop 8000 (Thermo Scientific, Rockford, IL) and electrophoresis through $0.8 \%$ agarose. A subset of 96 samples was chosen for GBS that were of high DNA quality and representative of 16 collection sites in Georgia and acquisitions from two collaborators.

DNA samples (250 ng) were digested with Mspl and Pst 1 at $37{ }^{\circ} \mathrm{C}$ for $2 \mathrm{~h}$ in a 96-well plate. Barcoded PstI adapters and MspI Y-adapters were ligated to the digested DNA fragments, as described in Qi et al., (2018). Small DNA fragments $(<400 \mathrm{bp})$ were eliminated using a Mag-Bind RxnPure Plus kit (Omega Bio-Tek). Polymerase chain reaction (PCR) was conducted with each sample individually using a barcode-specific forward primer and a common adapterspecific reverse primer using the following conditions: $95{ }^{\circ} \mathrm{C}$ for 30 seconds, then 16 cycles of $95^{\circ} \mathrm{C}$ for $30 \mathrm{~s}, 62^{\circ} \mathrm{C}$ for $20 \mathrm{~s}, 68^{\circ} \mathrm{C}$ for $15 \mathrm{~s}$, followed by $68{ }^{\circ} \mathrm{C}$ for $5 \mathrm{~min}$. Following cleanup with a Mag-Bind RxnPure Plus kit, PCR products were quantified by 
SYBR green fluorometry on a plate reader. The PCR products of the 96 samples were pooled (5 ng each), and the library was quantified using a Qubit 2.0. The GBS library was sequenced with an Illumina NextsEq. 500 mid output flow cell by the Georgia Genomics Facility (Athens, GA), generating single-end reads of $150 \mathrm{bp}$ in length.

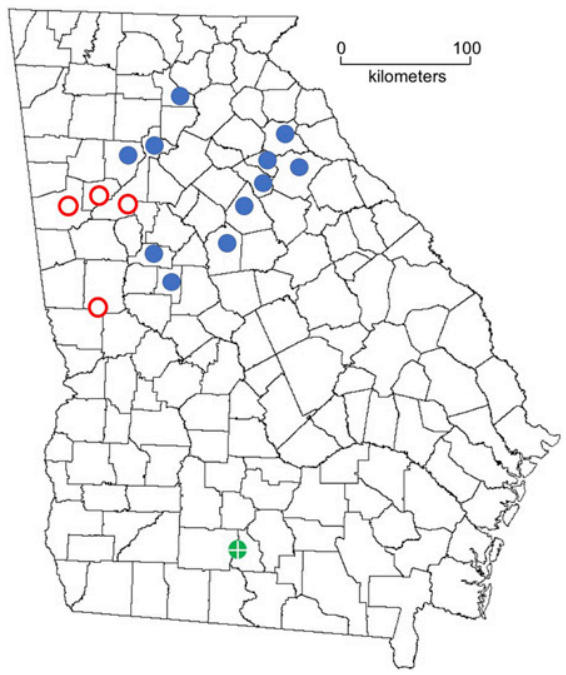

Fig. 1. Sites of Rhododendron canescens collection in Georgia. Leaf samples from at least four plants were collected per site. P1, filled blue circles; P2, open red circles; P3, cross-marked green circle.
Sequence data processing. FastQC (Leggett et al., 2013) was used to determine the quality of the sequence data. The sequence data were then processed using two software packages, STACKS v.1.44 (Catchen et al., 2013) and GBS-SNP-CROP (Melo et al., 2016). For STACKS, the raw sequence reads were filtered and trimmed to the length of $115 \mathrm{bp}$. STACKS analyses were performed using the following pipeline: process_radtags - ustacks - cstacks sstack - populations for diploid species, with 0.05 minor allele frequency. This generated a VCF file of the SNP matrix and initial population statistics.

For GBS-SNP-CROP analysis, raw GBS data were parsed to remove barcode sequences and cut sites and then trimmed using Trimmomatic (Bolger et al., 2014) to a uniform length (115 bp). The minimum phred score was set to 20 and the sliding window to $4 \mathrm{bp}$. Sequence reads were aligned using the Burrows-Wheeler Alignment tool ( $\mathrm{Li}$ and Durbin 2009) to a mock reference developed from $R$. canescens accession DA09. The binary matrix was generated and parsed using SAMtools (Li et al., 2009) in the downstream steps. Using the default settings for the diploid crop, SNP master matrix was generated followed by SNP calling.

Analysis of SNP data. The ancestral population clusters of $R$. canescens were established with the admixture model of STRUCTURE (Pritchard et al., 2000) 3 to 10 parallel Markov chains with a burn-in of 100,000 iterations and a run length of $1,000,000$ iterations following the burn-in. The STRUCTURE Harvester program was used to determine natural logarithms of probability data $[\mathrm{LnP}(\mathrm{K})]$ and the $\Delta \mathrm{K}$. STRUCTURE PLOT version 2.0 was used to create visual structure charts (Ramasamy et al., 2014). PCA was conducted in $R$ version 3.5.1 using the PCAdapt package (DuforetFrebourg et al., 2014) with 87 accessions that met threshold requirements. A weighted neighbor-joining tree was created in DARwin v6.0 using the default settings and data imported from the Genepop output file of STACKS (Supplemental Fig. 1).

The variant call file was used to manually make a numeric file with 0,1 , and 2 representing, respectively, homozygous reference alleles, heterozygous alleles, and homozygous alternate alleles. Nei statistics (Nei and Roychoudhury 1974) were calculated using $\mathrm{R}$ software (version 3.5.1; 2 July 2018) to estimate the genetic distance among the 87 accessions and the three population clusters determined by the PCA analysis. R was used to calculate all the population statistics using HierFstat (De Meeûs and Goudet 2007) and adegenet (Jombart 2008) function. This included gene diversity $\left(\mathrm{D}_{\mathrm{ST}}\right)$ and corrected gene diversity $\left(\mathrm{D}_{\mathrm{STP}}\right)$ among individuals, the overall gene diversity $\left(\mathrm{H}_{\mathrm{T}}\right)$ and corrected gene diversity $\left(\mathrm{H}_{\mathrm{TP}}\right)$ among populations, the fixation index $\left(\mathrm{F}_{\mathrm{ST}}\right)$ and corrected $\left(\mathrm{F}_{\mathrm{STP}}\right)$ based on population, and the inbreeding coefficient $\left(\mathrm{F}_{\mathrm{IS}}\right)$. The overall observed

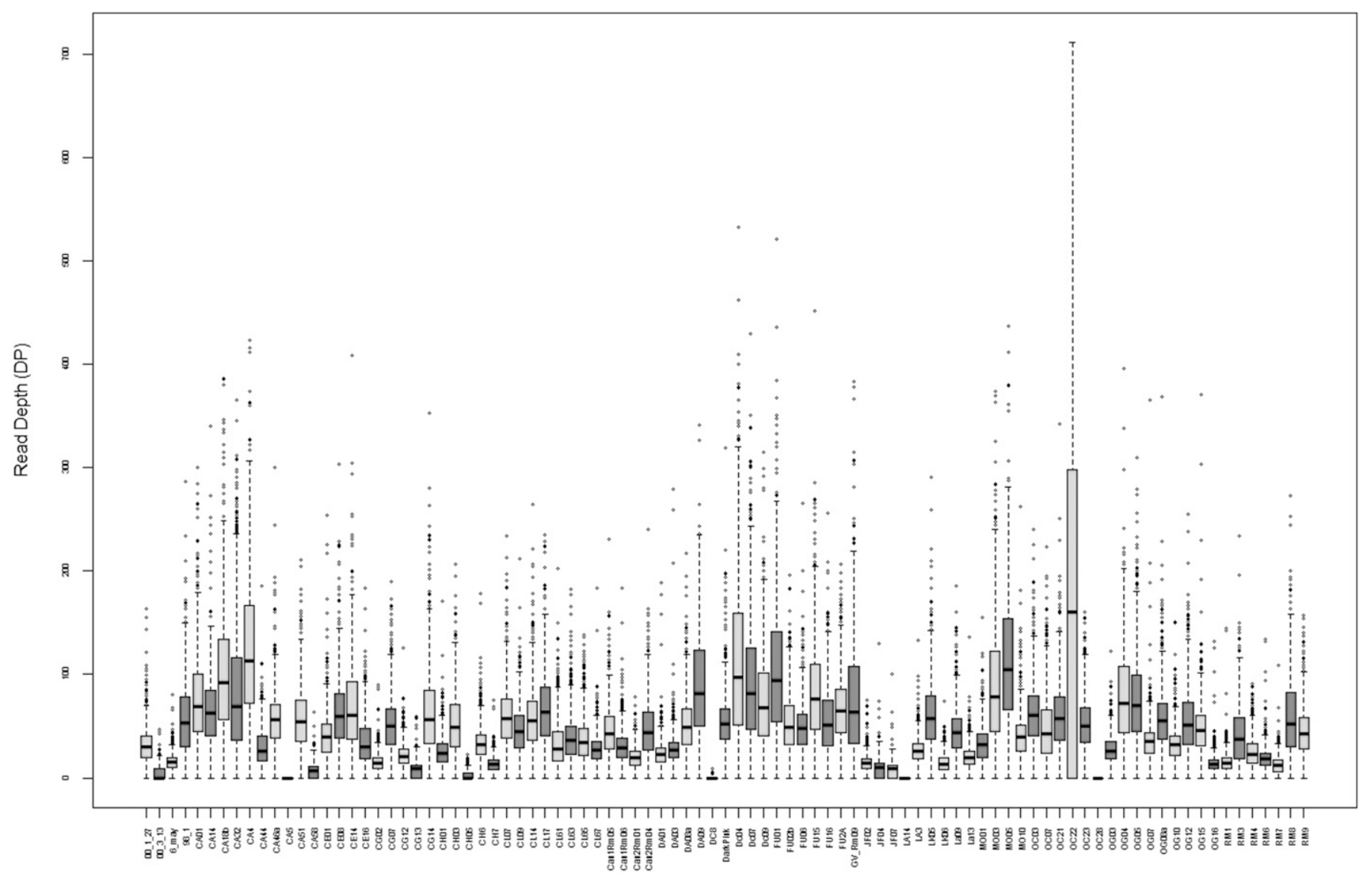

Fig. 2. Read depth of sequencing data. 
heterozygosity $\left(\mathrm{H}_{\mathrm{O}}\right)$ and genetic diversity $\left(\mathrm{H}_{\mathrm{S}}\right)$ within population was estimated based on mean allele frequency. $\mathrm{G}_{\mathrm{ST}}$, the proportion of species genetic diversity in relation to among-population variation, was calculated as $1-\left(\mathrm{H}_{\mathrm{S}} / \mathrm{H}_{\mathrm{T}}\right)$.

\section{Results}

GBS sequence data. Genetic analysis was conducted with $R$. canescens samples collected from 16 sites in Georgia (Fig. 1). Single-end sequencing of a GBS library of $96 R$. canescens accessions yielded 167,783,620 sequence reads. FastQC analysis indicated that the raw sequences were of good quality, with an average length ranging from 120 to $135 \mathrm{bp}$ and an average GC content of $46 \%$. The read depth count (Fig. 2) indicated an even coverage of the
$R$. canescens genome. After filtering, $57 \%$ of the sequences were retained as high-quality reads.

SNP identification and analysis. SNPs were identified and analyzed from $R$. canescens accessions with high-quality sequences and no missing data using STACKS and GBS-SNP-CROP software tools. A total of 3955 high-quality SNPs were called by STACKS from 91 accessions. These polymorphic sites comprised $0.85 \%$ of the total loci examined. The observed heterozygosity and homozygosity present in our GBS data were 0.1958 and 0.8042 , respectively. This matched the expected heterozygosity and homozygosity of 0.2557 and 0.7443 respectively. The inbreeding coefficient $\mathrm{F}_{\text {IS }}$ of the $R$. canescens lines was 0.2437 , indicative of an outcrossing population. All the statistics were calculated for variant sites.
In contrast to the de novo analysis of GBS data using STACKS, a reference-based analysis was conducted with GBS-SNP-CROP software. Because the $R$. canescens genome has not been sequenced, a mock reference was developed using GBS data of one of the accessions (DA09), and all other sample reads were aligned to this reference. After filtering, 3185 high-quality SNPs were called by GBS-SNP-CROP from 96 accessions.

Population structure analysis. The genetic structure of the $R$. canescens collection was examined through a STRUCTURE analysis of SNP data. Using GBS-SNP-CROP data, three populations $(\mathrm{P} 1-\mathrm{P} 3)$ were identified based on $\operatorname{LnP}(\mathrm{K})$ variance and delta $\mathrm{K}$ value (Fig. 3A and B). When STRUCTURE was conducted with STACKS data, two $R$. canescens populations were identified

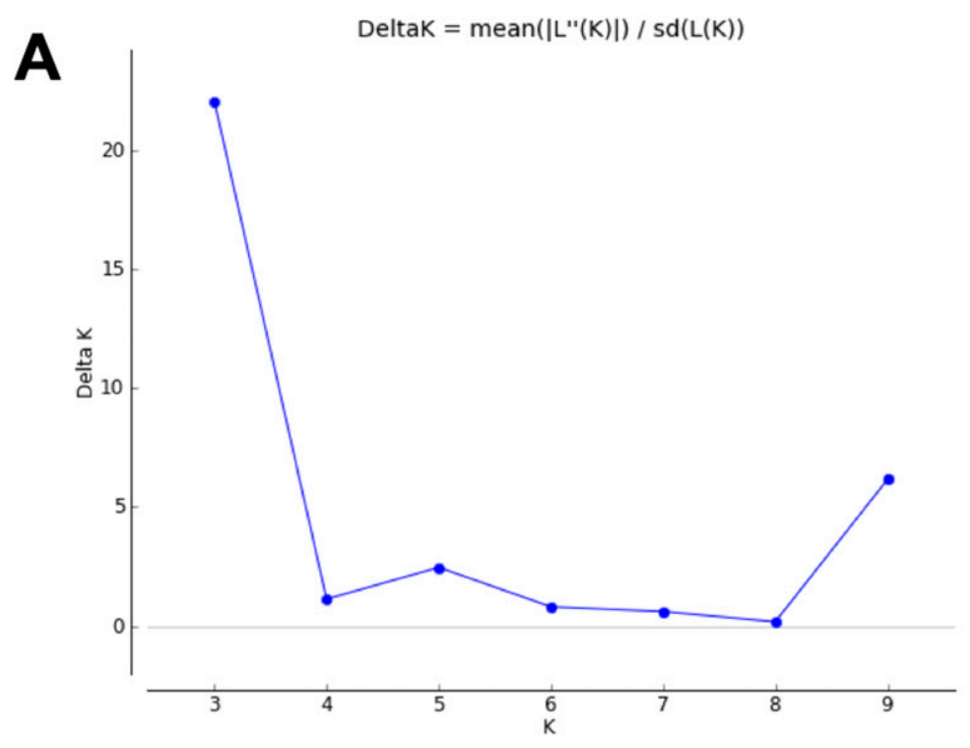

\section{B}
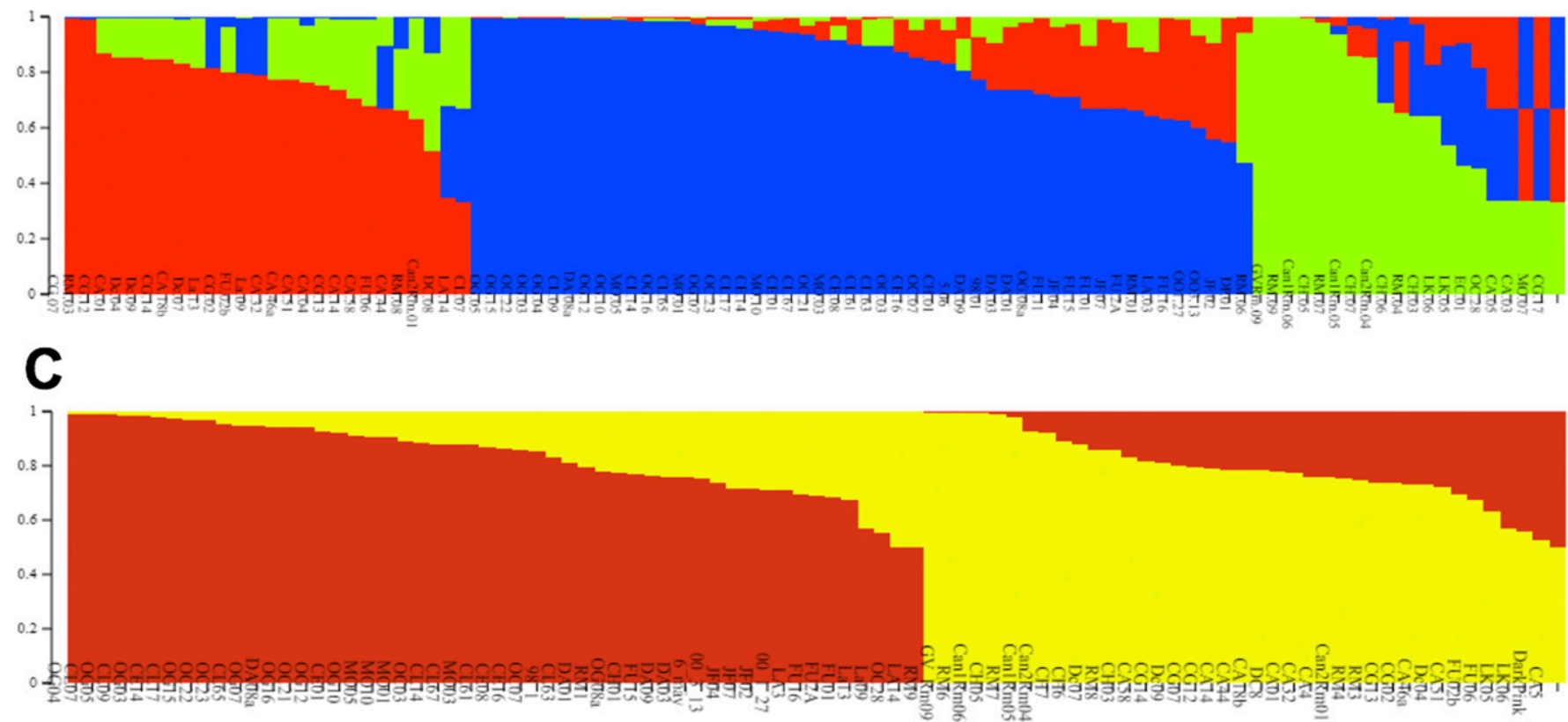

Fig. 3. STRUCTURE analysis. (A) Support for three optimal clusters based on delta K estimates from GBS-SNP-CROP data. (B) STRUCTURE results with GBSSNP-CROP data. (C) STRUCTURE results with STACKS data. 
(Fig. 3C). The larger group consisted of 52 azalea genotypes, and a smaller group had 39 genotypes. In light of PCA results that follow, the smaller group was reexamined by STRUCTURE, but there was no further differentiation (data not shown).

Principle component analysis. Genetic relationships within the $R$. canescens collection were further examined by PCA of SNP data. Three major clusters were observed among the 87 accessions using SNPs identified by STACKS analysis (Fig. 4) or GBSSNP-CROP analysis (not shown). The first and second principal components explained $\approx 12 \%$ of variance. The largest cluster contained samples from the central Georgia Piedmont, with the second largest containing samples from the western Georgia Piedmont region (Fig. 1). The third cluster was composed of samples from southern Georgia, northern Florida, and $R$. canescens samples of unspecified provenance from collaborators.

Population statistics. Genetic diversity was analyzed within and between the $R$. canescens populations (Table 1). The average heterozygosity within the species $\left(\mathrm{H}_{\mathrm{T}}\right)$ was 0.25 , and the average heterozygosity within populations $\left(\mathrm{H}_{\mathrm{S}}\right)$ was 0.24 . The proportion of species genetic diversity attributed to variation among populations $\left(\mathrm{G}_{\mathrm{ST}}\right)$ was calculated to be $4.0 \%$, a low value indicating significant gene flow within the species. Relatively low values were also observed for gene diversity among individuals $\left(\mathrm{D}_{\mathrm{ST}}\right)$ and the fixation index $\left(\mathrm{F}_{\mathrm{ST}}\right)$. The low $\mathrm{F}_{\mathrm{ST}}$ indicates that there was no significant differentiation between the populations and is consistent with $\mathrm{G}_{\mathrm{ST}}$ results.
The inbreeding coefficient $\left(F_{\text {IS }}\right)$ of the population under study is 0.2163 , a low value that would be expected with an outcrossing species. $\mathrm{F}_{\text {IS }}$ was positive, indicating that individuals within the population are more related than expected under a random mating model. Nei genetic diversity and genetic gain were also estimated among the $87 R$. canescens accessions and between the population clusters identified by PCA. This analysis found more variation among the individuals than between the three population clusters (Supplemental Table 2), in agreement with previous results.

\section{Conclusions}

A genotyping-by-sequencing approach was taken to characterize the genetic structure and diversity of a Rhododendron canescens germplasm collection. SNPs were identified by two software platforms, STACKS and GBS-SNP-CROP, and a genome-wide genetic variant file was developed. The genetic variation present in the germplasm collection was examined by STRUCTURE, a model-based Bayesian analysis, and PCA, a distance-based method. Taken together, these analyses indicated that there three population clusters present among the accessions analyzed.

The STRUCTURE results varied depending on whether SNP data from the GBS-SNPCROP or the STACKS pipeline were used. STRUCTURE with GBS-SNP-CROP and STACKS data identified three and two population clusters, respectively. These different outcomes may be because STACKS analysis involved de novo assembly, whereas GBS-
SNP-CROP was reference genome-based. The largest population cluster of 48 accessions (P1) was the same for both methods, but the remaining accessions were partitioned by STRUCTURE into two clusters of 22 accessions (P2) and 17 accessions (P3) when using GBS-SNP-CROP data. PCA of SNP data from GBS-SNP-CROP or STACKS supported the presence of three population clusters.

An analysis of $R$. canescens genetic diversity was included in a prior study (Chappell et al., 2008) that examined four $R$. canescens populations (six accessions each) with AFLP markers. Similar to that study, our investigation found a low $\mathrm{G}_{\mathrm{ST}}$ value, indicating that the proportion of diversity between populations was low, while the proportion of diversity within populations was high. Minimal differentiation between $R$. canescens populations was also indicated by the low $F_{\text {ST }}$ value. Chappell et al. (2008) suggested that this may be the result of gene flow between populations due to insect pollination. $R$. canescens is known to be pollinated by bumblebees, adrenid bees, butterflies, and hummingbirds. Populations P1 and P2 are geographically close, whereas accessions of P3 had more diverse origins. Introgression from other species of section Pentanthera may also have played a role in similarity found between populations.

Genetic markers, including SNPs and cpDNA loci, have been used to examine the genetic structure of a Japanese evergreen azalea species, $R$. indicum (Yoichi et al., 2018). SNPs were identified by multiplexed ISSR GBS (MIG-seq). Two genetically distinct lineages were detected, both of which
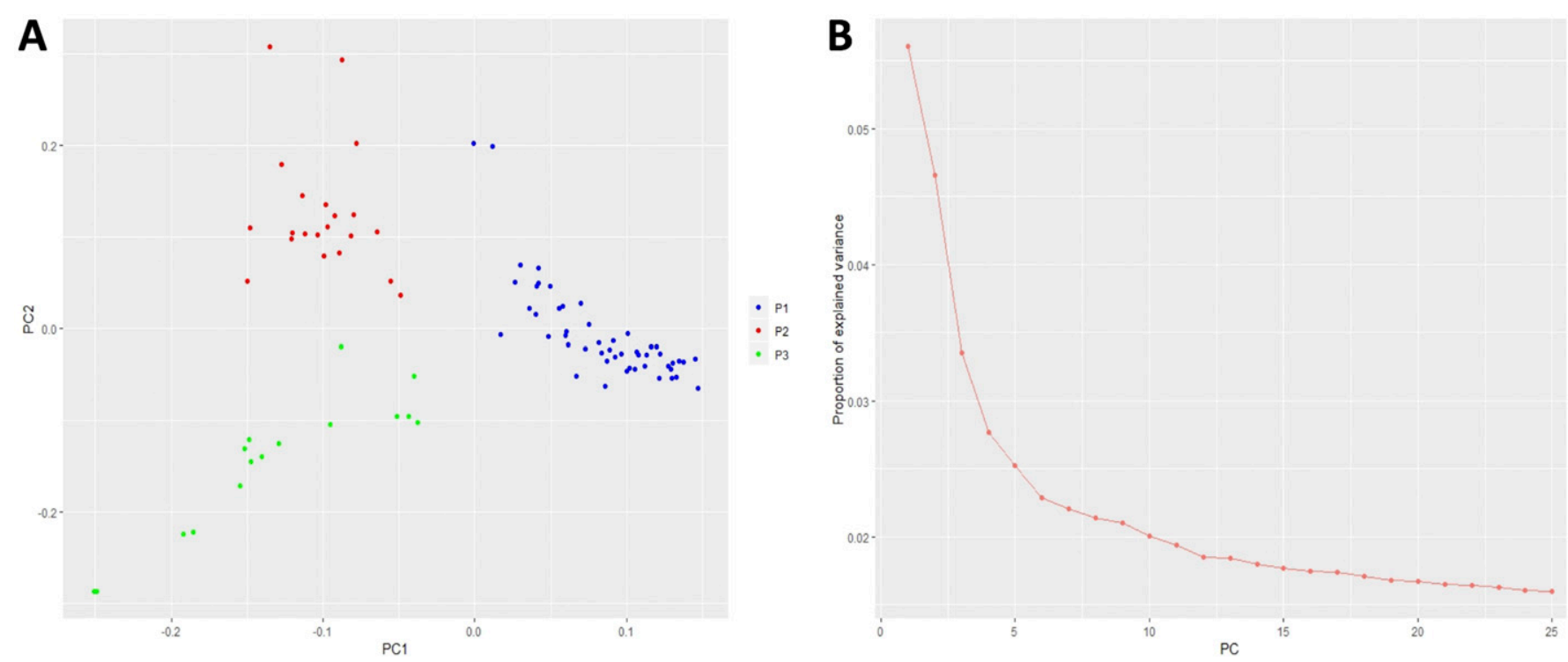

Fig. 4. Principal component analysis (PCA). (A) PCA with single nucleotide polymorphism data from STACKS pipeline. Populations P1, P2, and P3 as defined by STACKS analysis. (B) Proportion of variance explained by principal components.

Table 1. Genetic diversity statistics of STACKS data.

\begin{tabular}{|c|c|c|c|c|c|c|c|c|c|}
\hline$\overline{\mathrm{H}_{\mathrm{O}}}$ & $\mathrm{H}_{\mathrm{S}}$ & $\mathrm{H}_{\mathrm{T}}$ & $\mathrm{D}_{\mathrm{ST}}$ & $\mathrm{H}_{\mathrm{TP}}$ & $\mathrm{D}_{\mathrm{STP}}$ & $\overline{F_{S T}}$ & $\mathrm{~F}_{\mathrm{STP}}$ & $\mathrm{F}_{\mathrm{IS}}$ & $\mathrm{D}_{\mathrm{EST}}$ \\
\hline 0.1933 & 0.2452 & 0.2572 & 0.0120 & 0.2631 & 0.0180 & 0.0465 & 0.0682 & 0.2117 & 0.0238 \\
\hline
\end{tabular}


had DNA introgressed from geographically close populations of $R$. kaempferi. MIG-seq was also used to investigate rhododendron plants in a hybrid zone between two natural varieties of $R$. japonoheptamerum (Tamaki et al., 2017). SNP analysis distinguished the varieties and their hybrids and provided the basis for estimating that hybridization occurred 0.4 million years ago. In our investigation, GBS provided a cost-efficient means of generating SNP markers for genetic characterization an $R$. canescens germplasm collection. The high level of genetic diversity found within this collection indicates that screening for allelic variation in genes controlling architecture could be a viable approach to accelerate the breeding of plants with improved form.

\section{Literature Cited}

Bolger, A.M., M. Lohse, and B. Usadel. 2014. Trimmomatic: A flexible trimmer for Illumina sequence data. Bioinformatics 30:2114-2120.

Catchen, J., P.A. Hohenlohe, S. Bassham, A. Amores, and W.A. Cresko. 2013. Stacks: An analysis tool set for population genomics. Mol. Ecol. 22:3124-3140.

Chappell, M., C. Robacker, and T.M. Jenkins. 2008. Genetic diversity of seven deciduous azalea species (Rhododendron spp. section Pentanthera) native to the eastern United States. J. Amer. Soc. Hort. Sci. 133:374-382.

De Meeûs, T. and J. Goudet. 2007. A step-by-step tutorial to use HierFstat to analyse populations hierarchically structured at multiple levels. Infect. Genet. Evol. 7:731-735.

Duforet-Frebourg, N., E. Bazin, and M.G.B. Blum. 2014. Genome scans for detecting footprints of local adaptation using a Bayesian factor model. Mol. Biol. Evol. 31:2483-2495.

Galle, F.C. 1967. Native and some introduced azaleas for southern gardens: Kinds and culture. Amer. Hort. Mag. 46:13-23.
Jombart, T. 2008. adegenet: A R package for the multivariate analysis of genetic markers. Bioinformatics 24:1403-1405.

King, B.L. 1977. Flavonoid analysis of hybridization in Rhododendron sect. Pentanthera (Ericaceae). Syst. Bot. 2:14-27.

Kron, K.A. 1993. A revision of Rhododendron section Pentanthera. Edinb. J. Bot. 50:249-364.

Kron, K.A., L.M. Gawen, and M.W. Chase. 1993. Evidence for introgression in azaleas (Rhododendron; Ericaceae): Chloroplast DNA and morphological variation in a hybrid swarm on Stone Mountain, Georgia. Amer. J. Bot. 80:1095-1099.

Kurashige, Y., J.I. Etoh, T. Handa, K. Takayanagi, and T. Yukawa. 2001. Sectional relationships in the genus Rhododendron (Ericaceae): Evidence from matK and trnK intron sequences. Plant Syst. Evol. 228:1-14.

Leggett, R.M., R.H. Ramirez-Gonzalez, B.J. Clavijo, D. Waite, and R.P. Davey. 2013. Sequencing quality assessment tools to enable data-driven informatics for high throughput genomics. Front. Genet. 4:288.

Li, H. and R. Durbin. 2009. Fast and accurate short read alignment with Burrows-Wheeler transform. Bioinformatics 25:1754-1760.

Li, H., B. Handsaker, A. Wysoker, T. Fennell, J. Ruan, N. Homer, G. Marth, G. Abecasis, and R. Durbin. 1000 Genome Project Data Processing Subgroup. 2009. The sequence alignment/map format and SAMtools. Bioinformatics 25:20782079.

Mader, E., M. Shepherd, M. Vaughan, S. HoffmanBlack, and G. LeBuhn. 2011. Attracting Native Pollinators: Protecting North America's Bees and Butterflies. Storey Publishing, North Adams, MA.

Melo, A.T., R. Bartaula, and I. Hale. 2016. GBSSNP-CROP: A reference-optional pipeline for SNP discovery and plant germplasm characterization using variable length, paired-end genotyping-by-sequencing data. BMC Bioinformatics 17:1.

Nei, M. and A.K. Roychoudhury. 1974. Sampling variances of heterozygosity and genetic distance. Genetics 76:379-390.
Omernik, J.M. and G.E. Griffith. 2014. Ecoregions of the conterminous United States: Evolution of a hierarchical spatial framework. Environ. Mgt. 54:1249-1266.

Peterson, G.W., Y. Dong, C.C. Horbach, and Y.B. $\mathrm{Fu}$. 2014. Genotyping-by-sequencing for plant genetic diversity analysis: A lab guide for SNP genotyping. Diversity 6:665-680.

Pritchard, J.K., M. Stephens, and P. Donnelly. 2000. Inference of population structure using multilocus genotype data. Genetics 155:945-959.

Ramasamy, R.K., S. Ramasamy, B.B. Bindroo, and V.G. Naik. 2014. STRUCTURE PLOT: A program for drawing elegant STRUCTURE bar plots in user friendly interface. Springerplus 3:431.

Qi, P., D. Gimode, D. Saha, S. Schröder, D. Chakraborty, X. Wang, M.M. Dida, R.L. Malmberg, and K.M. Devos. 2018. UGbSFlex, a novel bioinformatics pipeline for imputation-free SNP discovery in polyploids without a reference genome: Finger millet as a case study. BMC Plant Biol. 18:117, doi: 10.1186/s12870-018-1316-3.

Scheiber, S.M., R.L. Jarret, C.D. Robacker, and M. Newman. 2000. Genetic relationships within Rhododendron L. section Pentanthera G. Don based on sequences of the internal transcribed spacer (ITS) region. Scientia Hort. 85:123-135.

Tamaki, I., W. Yoichi, Y. Matsuki, Y. Suyama, and M. Mizuno. 2017. Inconsistency between morphological traits and ancestry of individuals in the hybrid zone between two Rhododendron japonoheptamerum varieties revealed by a genotyping-by-sequencing approach. Tree Genet. Genomes 13:4.

Wang, Y., C.D. Robacker, and S.K. Braman. 1998. Identification of resistance to azalea lace bugs among deciduous azalea taxa. J. Amer. Soc. Hort. Sci. 123:592-597.

Yoichi, W., I. Kawamata, Y. Matsuki, Y. Suyama, K. Uehara, and M. Ito. 2018. Phylogeographic analysis suggests two origins for the riparian azalea Rhododendron indicum (L.) Sweet. Heredity 121:594-604. 
Supplemental Table 1. Location of $s$ lines used in GBS analysis.

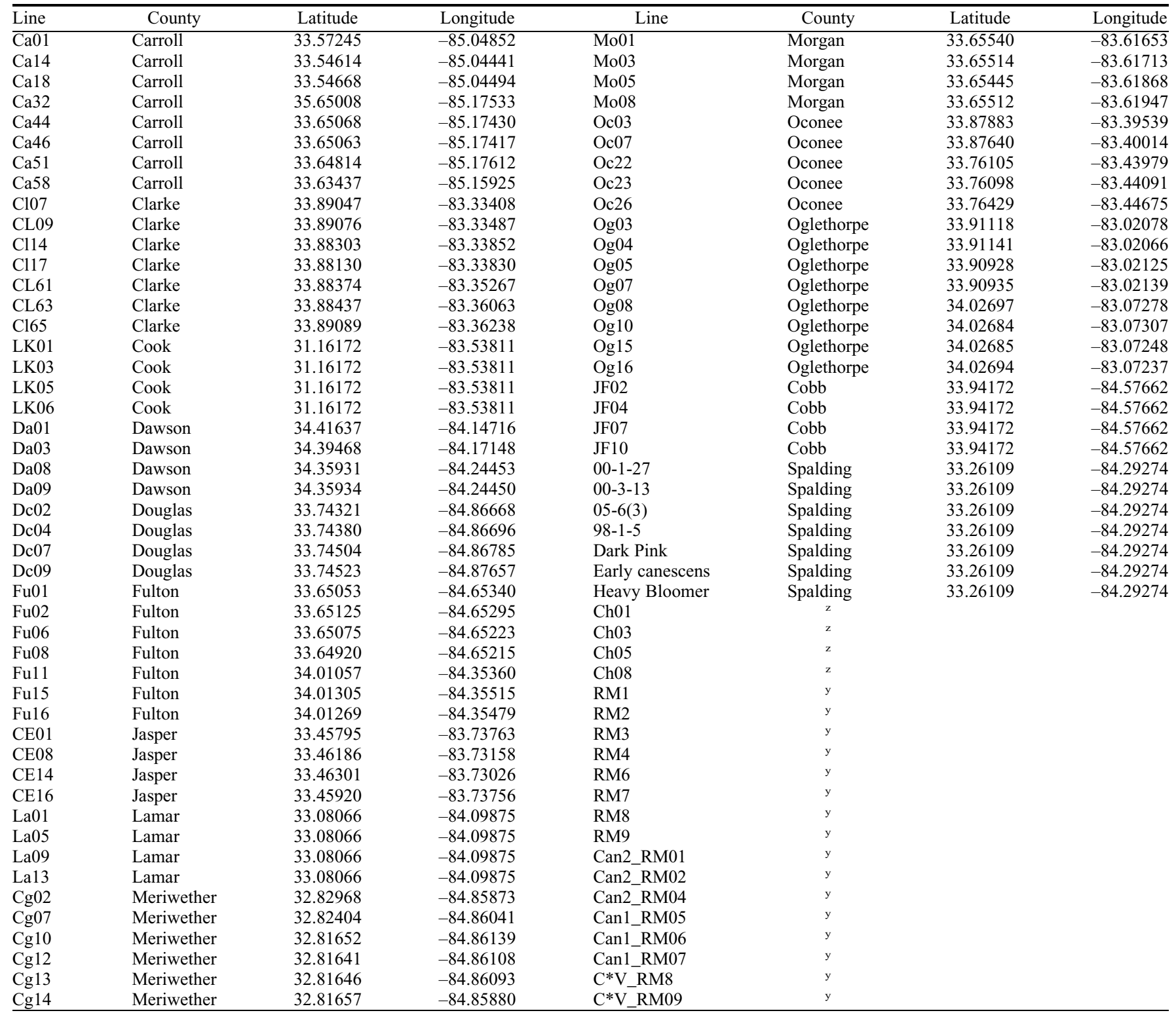

${ }^{\mathrm{z}}$ Accessions from collaborator 1 .

${ }^{\mathrm{y}}$ Accessions from collaborator 2 . 


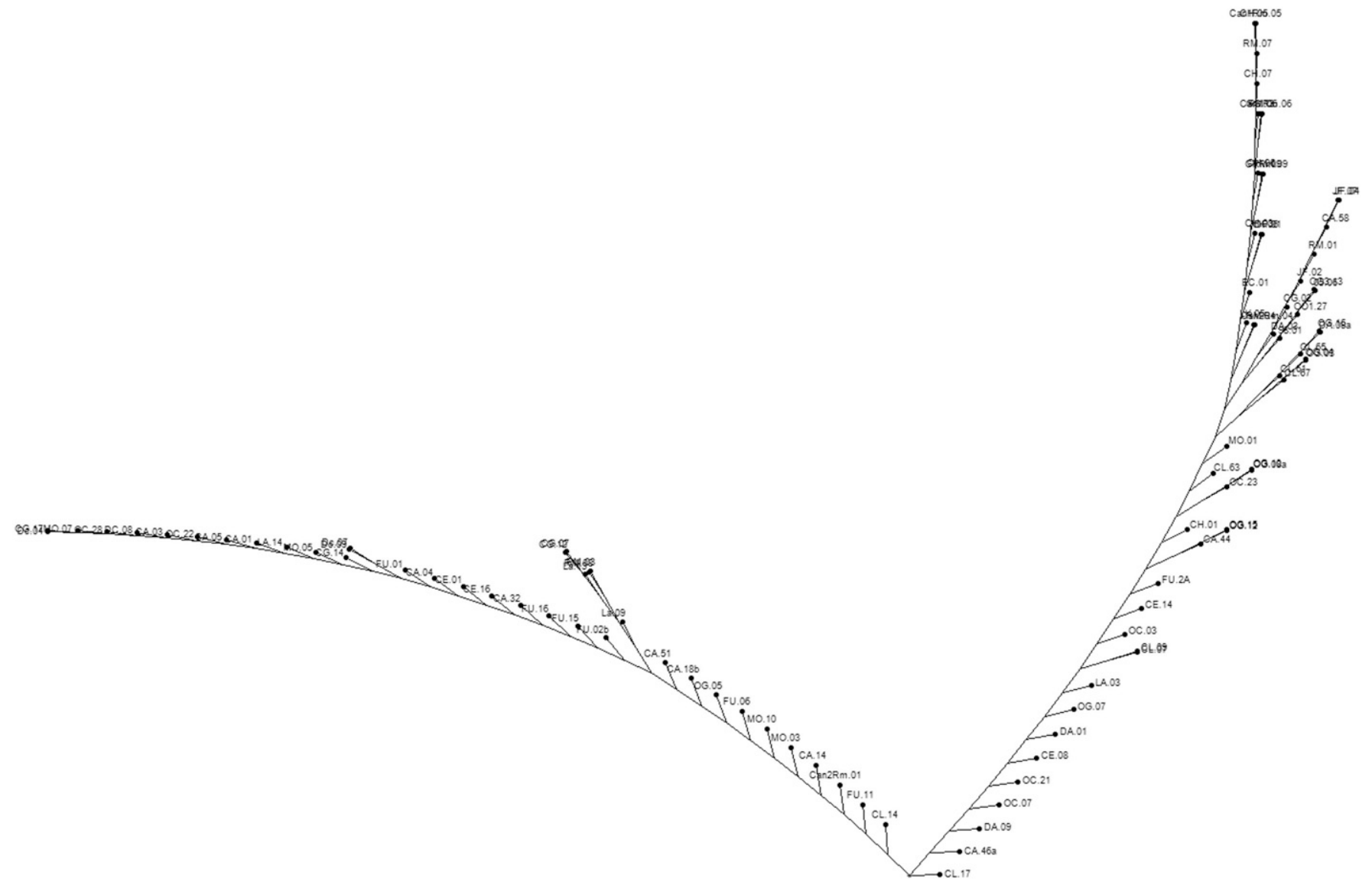

Supplemental Fig. 1. Phylogenetic relationships of Rhododendron canescens accessions. A weighted neighbor-joining phylogenetic tree created in DARwin v6.0. 